\title{
Integrating Real-Time Analytics and Continuous Performance Management in Smart Manufacturing Systems*
}

\author{
Senthilkumaran Kumaraguru, Boonserm (Serm) Kulvatunyou, and K.C. Morris
}

Systems Integration Division, Engineering Laboratory, NIST

Gaithersburg, MD, USA.

\{fns1, boonserm. kulvatunyou, kcm\}@nist.gov

\begin{abstract}
This paper proposes an approach to integrate real-time analytics with continuous performance management. The proposed system exploits the increasing availability of industrial process and production performance data. This paper identifies components of such a system and the interface between components within the system. The components presented in this paper form the basis for further research on understanding potential interoperability issues and required standardization efforts to support development of a system.
\end{abstract}

Keywords: performance management, smart manufacturing, continuous improvement, real-time analytics.

\section{Introduction}

Smart manufacturing (SM) systems are envisioned to have the ability to adapt to new situations by using real-time data for intelligent decision-making, as well as predicting and preventing failures proactively in real time. The nature of plant data today is characterized by large volume, large varieties, high velocity, low veracity (accuracy), uncertain validity, and volatility (timeliness). Data analytics can use this type of data to provide insights for the purpose of performance management. SM will need performance management systems that have the capability to generate and consume such data to continuously monitor, measure, and control performance in real time [1]. In this context, this paper presents an approach for integrating performance management of SM systems with real-time data analytics. Performance management systems employing such an approach enable continuous improvements to performance criteria such as costs, safety, and sustainability. Over time, the result of deploying such a performance management system will benefit manufacturers by providing a more dynamic response to performance concerns. This in turn will enable realizations of smart manufacturing systems that benefit end customers with low cost, sustainable, and customizable product choices [2]. The proposed integrated system for performance management is discussed in detail in the next section.

\footnotetext{
* The rights of this work are transferred to the extent transferable according to Title 17 U.S.C. $§ 105$.
} 


\section{Real-Time, Continuous Performance Management System}

Effective performance management is both reactive and proactive. Reactively, performance measurements drive two actions when there is a performance deviationdiagnosis and resolution. Diagnosis involves pinpointing potential problem areas, while the resolution involves finding and applying corrective actions. Proactively, when there is no deviation from a target performance for a period of time, the system can identify an opportunity for more improvements to the performance. In other words, manufacturers can and should continuously monitor and adjust target performance. Also proactively, performance management can be used to predict performance issues in real time.

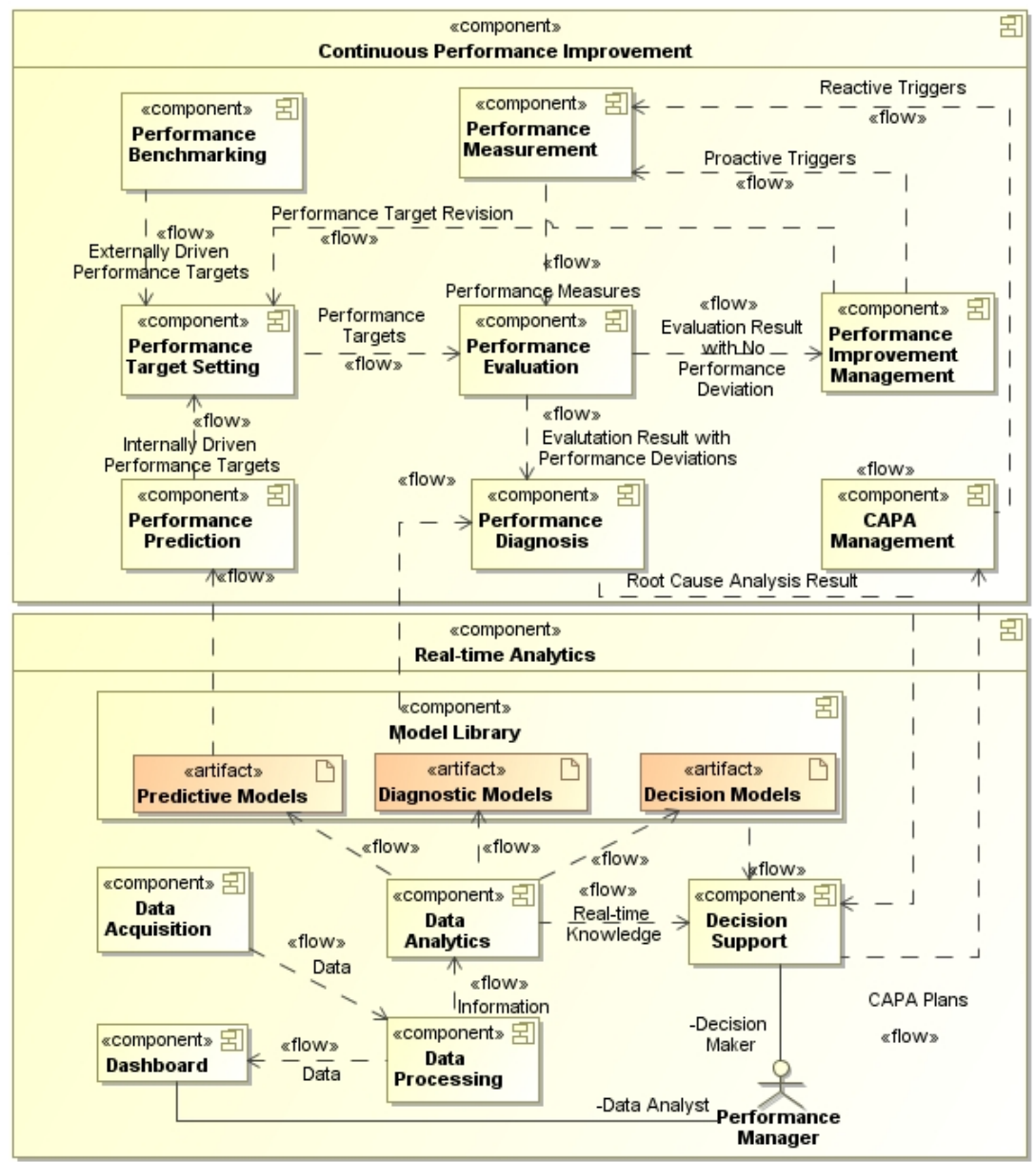

Fig. 1. Real-time analytics and continuous performance management system components 
These desired functionalities necessitate the following major characteristics in the system: 1) data needs to be available for an intelligent and informed decision in real time; 2) performance and exceptions need to be predicted regularly and evaluated against performance targets; 3 ) performance deviation needs to be continuously monitored and improved; and 4) performance diagnosis and resolution capabilities need to be available online and evolved over time.

Figure 1 highlights the major components of the proposed system. At the top level the system is divided into two components: Real-time Analytics and Continuous Performance Improvement. The Real-time Analytics component discussed in section 2.1, is responsible for turning data from the system into information that can guide decision making. It recommends corrective and preventive actions (CAPA) and produces analytical models that are used by the Continuous Performance Improvement component. The Continuous Performance Improvement component discussed in section 2.2, focuses on measuring and evaluating data against targets. Deviation from targets will cause diagnosis and resolution. The system components are identified by $<<$ components $>>$ and flow of information between the components are specified by $<<$ flow $>>$ and is marked by a dotted line in Figure 1 and the interactions of a performance manager with the components are specified by a solid line.

\subsection{Real-Time Analytics}

The main functions of the Real-time Analytics component are to: (1) gather data and convert it into useful knowledge and intelligence, and (2) develop predictive, diagnostic, and decision models. The models developed are maintained in a library as shown in Figure 1. This library is a growing knowledge base about the system based on past performance. The other components described below support both the construction and use of these models. The models are also used by the Continuous Performance Improvement component.

\section{Data acquisition and Data Processing}

The data used to calculate performance metrics is often available in multiple locations, in multiple formats, and from multiple systems. MTConnect [3] and Open Platform Communications (OPC) (previously Object Linking and Embedding for process control) Unified Architecture (UA) [4] are two extensible standards for exchanging shop floor data between systems. These standards enhance the utility and reduce computational complexity of mining unstructured information. Currently supported data types in these standards include measured physical quantities such as forces, acceleration, energy, flow rate, positional data, and clock time. The calculated data supported includes strain, torque, and wattage; and sensed events such as availability, error traces, emergency stops, part counts, and power state. This data needs further data cleaning and processing to deliver information to a system for modeling and decision making.

Figure 2 shows data flow between Data Acquisition, Data Processing, and Data Analytics. Unstructured data from a Human Machine Interface (HMI) may be processed by a Supervisory Control and Data Acquisition (SCADA) system, possibly followed by a Manufacturing Execution System (MES) system and by an Enterprise 
Resource Planning (ERP) system. Data for the manufacturing decision maker is available in a structured way through each of these systems typically with increasing levels of abstraction by aggregating the data. Historical data from these systems are used to generate performance predictions.

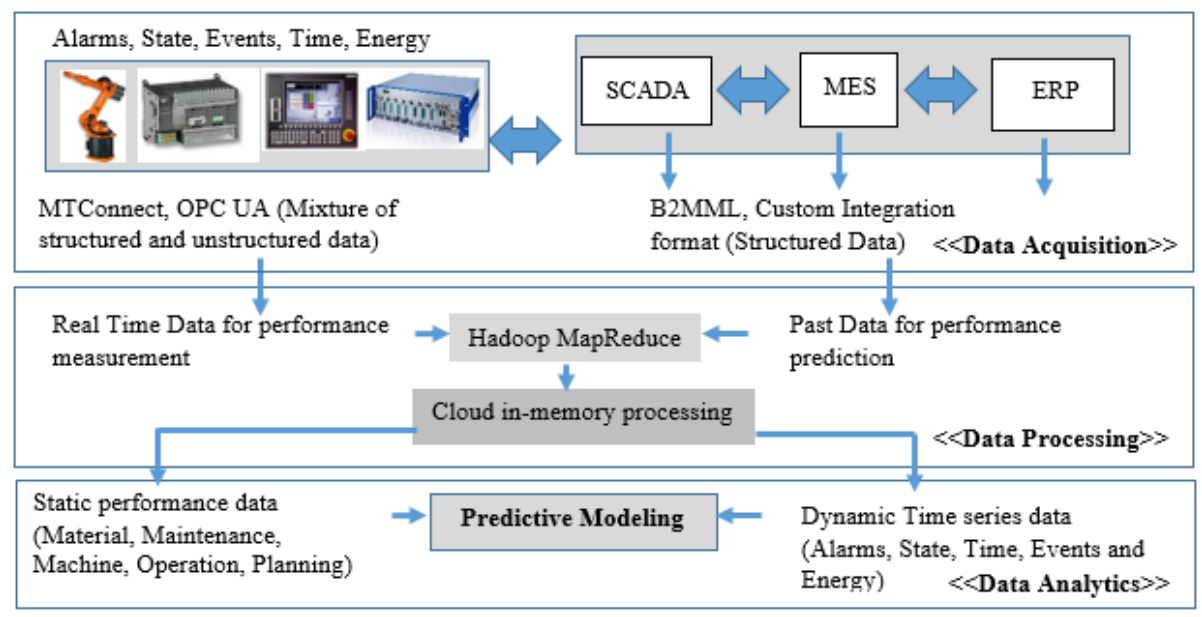

Fig. 2. Real-time data processing for performance management

Recent advances in processing capabilities dealing with large data sets through distributed data handling methods make it possible to extract useful, timely information from the unstructured data files. Data Processing shown in Figure 2 outlines the idea of using Hadoop for distributed processing to generate information from both structured and semi-structured data for real-time performance prediction. MapReduce [5] is a programming model for processing large data sets with a parallel, distributed algorithm on a cluster. Hadoop MapReduce is an open source implementation of this data processing programming model. Using this paradigm, a master node in a cluster takes $\log$ files as input and divides them into smaller sub-problems and distributes them into worker nodes. The worker nodes try to synthesize the information from the assigned sub problems by matching the codes. This is followed by a reduce step that collects the codes and combines the output in the form of a time-series of the actual events, alarms, and meta-data from the log files. The cloud in-memory processing provides the ability to connect to existing data sources and access to visually rich interactive dashboards. Easy navigation and ability to modify queries on the fly is an appealing factor to many users. Since these dashboards can be populated with fresh data, it allows users to have access to real time data and perform analytics quickly, which is a critical factor in real-time performance management.

\section{Data Analytics Model Library and Dashboard}

When dynamic time-series data is available, the Data Analytics component uses this information to generate prediction models for the chosen performance metrics. ARIMA (Autoregressive Integrated Moving Average) modeling methodology and GRACH 
(Generalized Autoregressive Conditional Heteroskedasticity) are popular statistical methods for prediction using time series data [6]. The multi-layer perceptron (MLP) with back propagation learning is a machine learning technique often employed in recent time-series studies [6]. Employing these methodologies in a plant or equipment prediction model, aids in goal settings for key performance metrics [7]. The Model Library component stores these prediction models for use by the Performance Prediction component. Similarly, diagnostic and decision models are also developed and stored for use by the Continuous Performance Improvement components.

Processed information is used to compute performance measures through the Performance Measurement component and visually present them in a Dashboard. The Dashboard component provides a visual interface that displays common key performance indicators to the end-user in a single view. For real-time data fed into the dashboard, the connections to the database and visualization modules are updated on a chosen frequency.

\section{Decision Support}

The Decision Support component uses the Model Library to help the decision maker choose corrective and preventive actions. The corrective action could be to choose a new set of operating parameters to deal with a problem situation or to change or modify the component of a physical resource or to adjust the production levels to suit a new control recipe. These decisions are targeted towards different stakeholders and are made available to the Continuous Performance Improvement component for dissemination.

\subsection{Continuous Performance Improvement}

The main functions of the Continuous Performance Improvement component are to: (1) measure performance, (2) evaluate performance against targets, and (3) recommend areas for improvement either through diagnosis of problems or identification of opportunities. The primary interaction with the Real-time Analytics component is through the library of analytics models. Performance Prediction uses the predictive models to set performance targets, revise forecasts and change input conditions. Performance Diagnosis uses diagnostic models to categorize exceptions and trigger exception handling that produces root cause analysis results. It interacts with the Decision Support subcomponent of Real-time Analytics to produce CAPA plans with assistance from the human Performance Manager. The CAPA plans are fed into the CAPA Management subcomponent to manage performance corrections, e.g., scheduling a preventive maintenance. After a corrective action it triggers Performance Measurement, which is followed by Performance Evaluation. The Performance Evaluation subcomponent compares measured performance to predicted performance, and closes the continuous performance monitoring and diagnosis loop by triggering Performance Diagnosis when it finds a deviation. When there are no deviations during an evaluation, the Performance Evaluation subcomponent triggers the Performance 
Improvement subcomponent to adjust performance targets if possible. The Performance Improvement subcomponent aims to maximize performance by revising performance predictions and targets and sending the recommended changes to different stakeholders. Performance Benchmarking develops and collects both internal and external benchmark data and helps set performance targets. The process will continue even when the system reaches a level where the system performance fails to improve over a certain period of time. The subcomponents shown in Figure 1 are described below.

\section{Performance Measurement and Evaluation}

The key performance indicators (KPIs) are identified among many performance measures and their definitions are modeled. The Performance Measurement subcomponent manages the KPI definitions and their data requirements and uses the Data Processing sub-component to compute the real-time KPIs for the predefined frequencies. The Performance Evaluation component compares the predicted KPI target values and current measured values to look for deviations.

\section{Performance Prediction, Benchmarking, and Target Setting}

The Performance Prediction subcomponent sets time periods and predicts thresholds for the performance targets using prediction models from the Real-time Analytics component. The prediction interval varies as the real-time data is added to update the prediction model. Performance data is systematically checked against the prediction model to look for performance problems.

Performance Benchmarking is used in setting targets that are driven by external drivers. The benchmarking process may make use of data collection from best of breed organizations through surveys, interviews, focused group and industry meetings, publications and site visits. Performance targets should be revised when performance measurement and prediction indicates an opportunity for improvement by comparing current performance with the benchmarks.

\section{Performance Diagnosis and CAPA Management}

Figure 3 shows details of how the Real-time Analytics and Continuous Performance Improvement components interact to diagnose performance issues. The left side of the Figure 3 depicts the Data Analytics subcomponent as related to diagnostic modeling, while the right side depicts the Performance Diagnosis subcomponent. During performance evaluation when a deviation from a predicted performance target is found, a performance diagnosis procedure is triggered. The essential function of Performance Diagnosis in the case of asset performance management as shown in Figure 3 is the root cause analysis. In the root cause analysis process, a Pareto analysis is carried out to separate the vital few problems from the trivial many. A weak point analysis followed by a criticality analysis is carried out to understand the severity of the problems and their root causes [8]. Based on the maintenance policy, suitable corrective and preventive actions are suggested. 


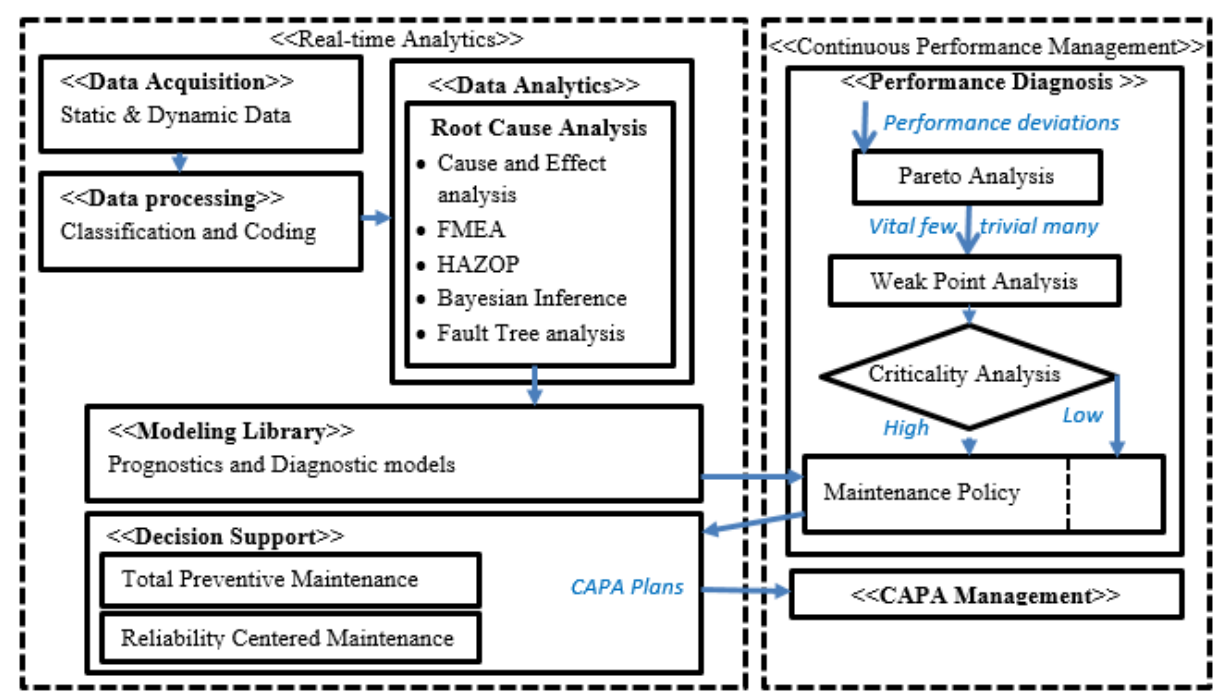

Fig. 3. Performance diagnosis and diagnostic modeling in case of asset performance management

The Data Analytics component shows different root cause analysis methods that may be used alone or in combination to develop diagnostic and prognostic models relevant to a performance management of assets as shown in Figure 3. Cause \& effect analysis, Failure mode effect analysis (FMEA), hazard and operability study (HAZOP) are the commonly used root-cause analysis methods used in the weak point analysis stage [9]. After the weak point analysis, a Bayesian inference and a fault tree analysis can be used in the criticality analysis stage. These analyses are part of the Data Analytics component identified in Figure 1.

\section{Continuous Improvement Management}

When the performance evaluations report no deviations in performance, the continuous improvement process is triggered. The continuous improvement process may follow a PDCA cycle (Plan, Do, Check, Act) or DMAIC (Define, Measure, Analyze, Improve, Control) approach to arrive at an incremental or breakthrough performance improvement [10]. The Performance Improvement sub-component also revises prediction algorithms and targets based on plans for introduction of new technologies, products, processes, and services.

\section{Conclusions and Future Work}

In this paper, an approach for utilizing data-driven models for a real-time, continuous performance management system has been proposed. The system is motivated by the smart manufacturing system vision in which adaptability and responsiveness are characterized as important metrics and capabilities for the next generation manufacturing 
systems. The proposed approach combines the use of state-of-art real-time data processing technologies recently available from the information technology community with predictive data analytics algorithms to form a continuous performance monitoring and improvement cycle thereby enabling real-time responsiveness. To that end, key components in the system are identified. Algorithms commonly used for producing predictive models are also discussed. As experience is gained with implementing this system, NIST will identify opportunities for standardizing interfaces to these components.

\section{Disclaimer}

Certain commercial products identified in this paper were used only for demonstration purposes. This use does not imply approval or endorsement by NIST, nor does it imply that these products are necessarily the best for the purpose.

\section{References}

1. Davis, J., Edgar, T., Porter, J., Bernaden, J., Sarli, M.S.: Smart manufacturing, manufacturing intelligence and demand-dynamic performance. Computers \& Chemical Engineering 47, 145-156 (2012)

2. SMLC, Implementing 21 st century smart manufacturing, Workshop summary report (2011)

3. Vijayaraghavan, A.: MTConnect for realtime monitoring and analysis of manufacturing enterprises. In Proceedings of the international conference on digital enterprise technology, Hong Kong (December 2009)

4. Mahnke, W., Leitner, S.H., Damm, M.: OPC unified architecture. Springer, Heidelberg (2009)

5. Dean, D., Ghemawat, S.: MapReduce: Simplified data processing on large clusters. In: Proceedings of OSDI (2004)

6. Box, E.P.G., Jenkins, M.J., Reinsel, G.C.: Time series analysis: forecasting and control, 4th edn. John Wiley \& Sons, Inc. New Jersey (2014)

7. Shin, S.-J., Woo, J., Rachuri, S.: Predictive analytics model for power consumption in manufacturing. Procedia CIRP 15, 153-158 (2014)

8. Lei, et al.: Asset management. Springer, New York (2012)

9. Latino, R.C., Latino, K.C., Latino, M.A.: Root cause analysis: Improving performance for bottom-line results, 4th edn. CRC Press, USA (2013)

10. Sokovic, M., Pavletic, D., Pipan, K.K.: Quality improvement methodologies - PDCA cycle, RADAR Matric, DMAIC and DFSS. Journal of Achievements in Materials and Manufacturing Engineering 43(1), 476-483 (2010) 